\title{
Alternation of the dynamic coefficients of short journal bearings due to wear
}

\author{
Michael G. Papanikolaou', Michael G. Farmakopoulos ${ }^{2}$, Chris A. Papadopoulos, ${ }^{2, *}$ \\ ${ }^{1}$ School of Engineering \\ Department of Engineering Physics \\ Cranfield University, MK43 0AL, United Kingdom \\ ${ }^{2}$ Machine Design Laboratory \\ Mechanical Engineering Department \& Aeronautics \\ University of Patras, Patras-26504, Greece
}

\begin{abstract}
Wear in journal bearings occurs when the operating conditions (high load, high temperature, low angular velocity or low viscosity), downgrade the ability of the bearing to carry load. The wear depth increases because the rotor comes in contact with the bearing surface. Wear in journal bearings affects their characteristics because of its influence on the thickness of the fluid film. This influence can be detected in the dynamic behavior of the rotor and especially in the dynamic stiffness and damping coefficients.

In this paper, the effect of wear on the rotordynamic stiffness and damping coefficients ( $K$ and $C$ ) of a short journal bearing is investigated. $\mathrm{K}$ and $\mathrm{C}$ in this work are estimated by using two methods: a) a semi analytical method and b) finite element analysis implemented in the ANSYS software. The main goal of this research is to make the identification of wear in journal bearings feasible by observing the alternation of their dynamic coefficients. Both of the methods implemented are proven to be useful, while finite element analysis can provide more accurate results.
\end{abstract}

Keywords: hydrodynamic journal bearing, wear, dynamic coefficients

Nomenclature:

$\begin{array}{llll}C_{i j}\left(\mathrm{~N}^{*} \mathrm{~s} / \mathrm{m}\right) & : \text { Damping dynamic coefficients } & R(\mathrm{~m}) & : \text { Bearing radius } \\ c(\mathrm{~m}) & : \text { Clearance } & \mathrm{r}, \mathrm{t} & : \text { Rotating coordinate system } \\ D(\mathrm{~m}) & : \text { Bearing diameter } & S & : \text { Sommerfeld number } \\ d_{0}(\mathrm{~m}) & : \text { Wear depth } & W(\mathrm{~N}) & : \text { Axial load } \\ e_{0}(\mathrm{~m}) & : \text { Journal eccentricity } & \varepsilon(\mathrm{m}) & : \text { Ecentricity ratio } \\ F_{i}(\mathrm{~N}) & : \text { Forces } & \Theta, \theta(\mathrm{rad}) & : \text { Peripheral coordinates } \\ F_{0}(\mathrm{~N}) & : \text { Bearing load } & \mu\left(\mathrm{N}^{*} \mathrm{~s} / \mathrm{m}\right) & : \text { Oil viscosity } \\ h(\mathrm{~m}) & : \text { Lubricant thickness } & \sigma & : \text { Modified Sommerfeld number } \\ K_{i j}(\mathrm{~N} / \mathrm{m}) & : \text { Stiffness dynamic coefficients } & \varphi_{0}(\mathrm{rad}) & : \text { Attitude angle } \\ L(\mathrm{~m}) & : \text { Bearing length } & x, y & : \text { Inertial coordinate system } \\ P(\mathrm{~Pa}) & : \text { Hydrodynamic pressure } & \Omega(\mathrm{rad} / \mathrm{sec}) & : \text { Angular velocity }\end{array}$

\section{Introduction}

The response of a rotor-bearing system depends on the geometric and material characteristics of the rotor, the exciting forces and the dynamic coefficients of the supporting journal bearings. The lubrication of the supporting bearings is described by the hydrodynamic theory expressed by the Reynolds equation.

A usual situation in such systems is wear occurring on the bearing surface. Wear arises due to overload or due to dry friction during startup or shutdown of the system, leading to contact between

\footnotetext{
* Corresponding author
} 
the rotor and the bearing surface. In such cases, wear affects the oil thickness and subsequently the pressure distribution. Moreover, the equilibrium point of the rotor, the dynamic coefficients of the bearing and the response of the system are affected as well.

The onset and development of wear in plain hydrodynamic journal bearings under repeated stop/start cycles have been studied experimentally by Mokhtar et al. [1]. The wear that occurred was easily discernable; however, localized changes in diametric clearance, surface finish and roundness of the bearing bore were measured after various numbers of operating cycles had been completed. A study of the wear location within the bearings showed that it was caused entirely by the sliding motion that occurred during start up and that no significant contribution to the wear process was observed at shutdown. This group also observed that, after an initial rapid phase of wear was completed, the surface finish of the hardened steel shaft was reproduced in regions of the surface of the bearing subjected to continued wear.

Kumar and Mishra [2] investigated numerically the effects of geometric change due to wear on the stability of hydrodynamic turbulent journal bearings, following Constantinescu's turbulent lubrication theory. They drew stability curves for various values of wear depth parameter, considering turbulence. Wear causes the stability of the rotor to deteriorate in the case of lightly loaded bearings. In the case of worn bearings, a lower L/D ratio gives better stability. Kumar and Mishra also concluded in [3] that increasing wear, causes the decrease of the load carrying capacity. Additionally, the frictional drag and the flow rate of a journal bearing operating under turbulent flow conditions are increased. The effect of wear on load carrying capacity and friction is greater at lower L/D ratios.

Laurant and Childs[4] performed tests which showed that in a plugged-orifice bearing there were increases in direct and cross-coupled stiffnesses and direct damping at higher values of eccentricity ratios. No similar increases were observed with worn bearings. Load capacity was not significantly influenced by large simulated wear on the land. The test results indicated that neither a single plugged orifice nor significant wear on the bearing land would disable a well-designed hybrid bearing.

Awasthi et al.[5] performed an analysis of a non-recessed worn hybrid journal bearing with orifice restrictors. They used the Newton-Raphson method (FEM) to solve the Reynolds equation for the flow of the lubricant in the bearing clearance space. The results showed that the wear affects the bearing performance significantly. The found that: (i) for a specified load and restrictor design parameter the changes in the performance characteristic parameters were more pronounced at higher values of the wear depth parameter (ii) due to significant variation in the performance characteristic parameters, the effect of wear should be considered for running a system satisfactorily over a designed life span.

Vaidyanathan et al.[6] studied the characteristics of noncircular bearings, considering the effects of turbulence and cavitation. They considered four bearing profiles: circular, worn-circular, two-lobe and elliptical. The linearized lubrication theory of $\mathrm{Ng}$ and Pan was used to simulate the turbulence. Reynolds equation was suitably modified to account for both turbulence and cavitation. The equation was incorporated into the Elrod cavitation algorithm. This numerical procedure implicitly incorporated the JFO boundary conditions at rupture and reformation boundaries.

Hashimoto et al [7] presented the effect of geometric change due to wear on the dynamic characteristics of journal bearings. They analyzed the dynamic characteristics, such as spring and damping coefficients, of a rigid rotor supported by two identical symmetrically aligned bearings for various wear depth parameters. They concluded that the geometric change due to wear has significant effects on the principal spring coefficients and the cross-coupled damping coefficients.

In a recent study Chasalevris et al. [8] studied the dynamic effect of bearing wear on the rotorbearing response. The Rayleigh coupled equations were used to describe the vibration of the rotor while the journal bearing forces have been evaluated by solving the Reynolds equation. They concluded that bearing wear affects the dynamics of the rotor introducing additional half-harmonics: $1 / 2 \mathrm{X}, 3 / 2 \mathrm{X}, 5 / 2 \mathrm{X}$ etc. The $1 / 2 \mathrm{X}$ harmonic is more sensitive when wear is developed and the journal whirling is rather large.

An earlier attempt to connect the wear depth with the dynamic characteristics is presented by Gertzos et al. [9]. They used a Computational Fluid Dynamics (CFD) analysis to solve the NavierStokes equations of a worn hydrodynamic journal bearing. They concluded that all the presented bearing characteristics are significantly altered as wear depth increases. Eccentricity and attitude angle increase as the side oil flow and the friction coefficient decrease.

Rozeanu and Kennedy [10] found that wear occurs in three different locations in journal bearings, with each of the locations showing a different predominant wear mechanism. Finally, they showed 
that the study of the wear problem requires a different model experiment for each of the three different wear modes, and each wear mode also requires a different remedy to achieve wear reduction.

Ronen and Malkin [11] investigated wear mechanisms in hydrodynamic bearings by contaminant abrasive particles in the oil film. This type of wear is one of the main factors responsible for the failure of hydrodynamic bearings especially when operating in dusty environments. They have conducted experiments with two shaft materials and three liner materials, giving a total of six material combinations. As a result, they deduced that a smaller hardness ratio resulted in relatively more liner wear and less shaft wear.

Li et al [12] studied the oil film pressure of a hydrodynamic bearing, the stress of bearing bush alloy, the simulation movement of the rotor, the reasons of failure and the wear mechanism of bearing bush in the Ansys software.

Bouyer et al [13] focused on two-lobe journal bearings. These mechanical components tend to be subjected to numerous startups and stops. During transient periods, direct contact between the journal and the bearing induces high friction in the lubricated contact and hence wear of the lining. They presented experimental data subjected to numerous starts and stops. Then, a comparison was made between the measured bearing performance and numerical results. They observed that hydrodynamic pressure increases, whereas the temperature at the film/bush interface slightly decreases on both the upper and lower lobes.

Phalle et al.[15] studied the influence of wear on the performance of a membrane compensated 2lobe four-pocket hybrid journal bearing system. The modified Reynolds equation governing the flow of lubricant in the clearance space of a journal bearing system was solved using FEM and NewtonRaphson methodand The numerically simulated results clearly indicated that the performance of the bearing wass greatly affected by wear.

Scharrer et al.[16] solved the Reynolds equation the turbulent flow of liquid hydrogen through an orifice compensated hydrostatic bearing with a worn stator element. The clearance function for the worn bearing was defined by the depth and circumferential location of wear and the resulting intersection of the journal and housing radii. The results showed that the performance of the bearing degrades steadily for wear amounts greater than 5 percent of the radial clearance and is relatively insensitive to the geometrical location of the wear.

Nikolakopoulos et al. [17] presented an analytical model in order to find the relationship among the friction force, the misalignment angles and wear depth. The Reynolds equation was solved numerically and the friction force was calculated in the equilibrium position. The friction coefficient was presented versus the misalignment angles and wear depths for different Sommerfeld numbers, thus creating friction functions dependent on misalignment and wear of the bearing. The variation in power loss of the rotor-bearing system was also investigated and presented as a function of wear depth and misalignment angles.

In this study, the well-known Dufrane [18] wear model has been implemented to introduce wear into the mathematical model. The model of a short bearing has been investigated. The oil forces acting on the rotor are analytically calculated and presented in closed form for the intact and the worn case. The same method has been applied for the dynamic stiffness and damping coefficients. The model has been validated using a finite element (FE) model of a hydrodynamic journal bearing using the ANSYS software. The dynamic coefficients are presented versus the wear depth parameter.

The purpose of this work is to give closed form expressions for the rotor dynamic coefficients (stiffness and damping), for short worn journal bearings or for worn journal bearings with a low $L / D$ ratio, where the wear effect is greater, according to Kumar and Mishra [1,2]. The second objective is to validate these closed form expressions using the CFD capability of ANSYS software. Then, the stiffness and damping coefficients versus the wear depth parameter will be presented and validated numerically.

\section{Wear model}

In this paragraph, the wear model that has been used is introduced. The wear model in this project was proposed by Dufrane [10] and it is shown schematically in figure 1 .

The film thickness $h_{0}$ in the non-worn region ( $\theta \leq \theta_{s}$ and $\left.\theta \geq \theta_{f}\right)$ is given by the equation:

$$
h_{0}=c+e_{0} \cdot \cos \theta
$$


where $c$ is the radial clearance of the journal bearing.

The starting and final points of the worn region $\left(\theta_{s}\right.$ and $\left.\theta_{f}\right)$ are given by the solution of the equation:

$$
\cos \left(\theta+\varphi_{0}\right)=\frac{d_{0}}{c}-1
$$

The lubricant thickness $h_{0}$ in the worn region $\left(\theta_{s} \leq \theta \leq \theta_{f}\right)$ is described by the equation:

$$
h_{0}=d_{0}+e_{0} \cdot \cos \theta-c \cdot \cos \left(\theta+\varphi_{0}\right)
$$

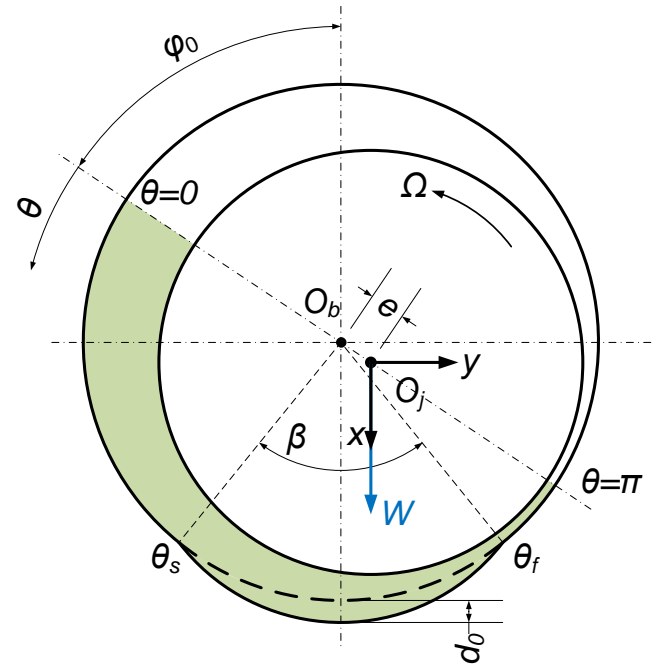

Figure 1: Worn journal bearing

\section{Analytical solution}

For the purposes of the present analysis, an input script was created in the Matlab software. Its basic functions are presented below:

- Determination of the geometrical and operational characteristics (eccentricity and attitude angle).

- Calculation of the dynamic coefficients of a non-worn journal bearing in the rotating coordinate system.

- Transformation of the dynamic coefficients from the rotating to the Cartesian coordinate system.

- Estimation of the relationship between the attitude angle and eccentricity in worn journal bearings within a loop.

- Estimation of the dynamic coefficients of a worn journal bearing in the rotating and Cartesian coordinate system.

Note: Intact (non-worn) journal bearings have not been considered as a special case, but as worn journal bearings with wear depth equal to zero $\left(d_{0}=0\right)$.

The mathematical background and the method implemented in the Matlab script are presented in the following lines.

The Reynolds equation, which describes the flow in journal bearings, has been used in the analytical solution [11]:

$$
\frac{1}{R^{2}} \frac{\partial}{\partial \theta}\left(\frac{\rho}{12} \frac{h^{3}}{\mu} \frac{\partial P}{\partial \theta}\right)+\frac{\partial}{\partial z}\left(\frac{\rho}{12} \frac{h^{3}}{\mu} \frac{\partial P}{\partial z}\right)=\frac{\partial}{\partial t}(\rho h)+\frac{\Omega}{2} \frac{\partial}{\partial \theta}(\rho h)
$$

The assumptions needed to obtain the solution of the Reynolds equation are [2]: 
- The amplitude motions around the equilibrium position have to be small, so $e(t)=e_{0}+\Delta e(t)$ and $\varphi(t)=\varphi_{0}+\Delta \varphi(t)$, where $\Delta e$ and $\Delta \varphi$ are small displacement quantities, respectively.

- Products of small quantities such as $\Delta e \cdot \Delta \varphi$ should be considered equal to zero.

- In the case of short journal bearings, the circumferential flow is very small, i.e. $\frac{\partial P}{\partial \theta} \cong 0$. As a result, the Reynolds equation reduces to:

$$
\frac{\partial}{\partial z}\left(\frac{\rho}{12} \frac{h^{3}}{\mu} \frac{\partial P}{\partial z}\right)=\frac{\partial}{\partial t}(\rho h)+\frac{\Omega}{2} \frac{\partial}{\partial \theta}(\rho h)
$$

where $h$ can be obtained by the equations (1) or (3) depending on the value of the angle $\theta$.

According to the assumptions made, the film thickness for the non-worn region can be written as:

$$
h_{0}=c+e_{0} \cdot \cos \theta=c+\left(e_{0}+\Delta e\right) \cdot \cos \left(\Theta-\varphi_{0}-\Delta \varphi\right)
$$

Considering that $\theta=\Theta-\varphi_{0}, \sin (\Delta \varphi) \cong \Delta \varphi$ and $\cos (\Delta \varphi) \cong 1$, equation (6) reduces to:

$$
h=\mathrm{c}+e_{0} \cdot \cos \theta+\Delta e \cdot \cos \theta+e_{0} \cdot \Delta \varphi \cdot \sin \theta
$$

The film thickness for the worn region can be written as:

$$
\begin{aligned}
& h_{0}=d_{0}+e_{0} \cdot \cos \theta-c \cdot \cos \left(\theta+\varphi_{0}\right)= \\
& =d_{0}+\left(e_{0}+\Delta e\right) \cdot \cos \left(\Theta-\varphi_{0}-\Delta \varphi\right)-c \cdot \cos \left(\theta+\varphi_{0}\right)
\end{aligned}
$$

Considering that $\theta=\Theta-\varphi_{0}, \sin (\Delta \varphi) \cong \Delta \varphi$ and $\cos (\Delta \varphi) \cong 1$, equation (8) reduces to:

$$
h_{0}=d_{0}+e_{0} \cdot \cos \theta-c \cdot \cos \left(\theta+\varphi_{0}\right)+e_{0} \cdot \sin \theta \cdot \Delta \varphi+\cos \theta \cdot \Delta e
$$

Integrating equation (5) two times, gives:

$$
P=\frac{6 \mu}{c^{3} H^{3}}\left[\begin{array}{l}
\left(e_{0} \cdot \Delta \dot{\varphi}-\frac{\Omega}{2} \cdot e_{0}-\frac{\Omega}{2} \cdot \Delta e\right) \cdot \sin \theta+\left(\Delta \dot{e}+\frac{\Omega}{2} \cdot e_{0} \cdot \Delta \varphi\right) \cdot \cos \theta+ \\
+\frac{\Omega}{2} \cdot c \cdot \sin \left(\theta+\varphi_{0}\right)
\end{array}\right] \cdot\left(z^{2}-\frac{L^{2}}{4}\right)
$$

The radial and tangential coefficients of the film force can be obtained by the following equation:

$$
\left[\begin{array}{l}
F_{r} \\
F_{t}
\end{array}\right]=2 \cdot \int_{0}^{L / 2} \int_{0}^{\pi} P(\theta, z, t) \cdot R \cdot\left(\begin{array}{c}
\cos \theta \\
\sin \theta
\end{array}\right) d \theta d z
$$

The area in which the film pressure is positive $(0<\theta<\pi)$ can be divided into 3 sub-areas:

a. $0<\theta<\theta_{s}$. In this area, the film thickness is equal to: $h_{0}=c+e_{0} \cdot \cos \theta$

b. $\theta_{s} \leq \theta \leq \theta_{f}$. In this area, the film thickness is equal to: $h_{0}^{\prime}=d_{0}+e_{0} \cdot \cos \theta-c \cdot \cos \left(\theta+\varphi_{0}\right)$

c. $\theta_{f}<\theta<\pi$. In this area, the film thickness is equal to: $h_{0}=c+e_{0} \cdot \cos \theta$

The form of the solution of equation (11) is: 


$$
\left[\begin{array}{c}
F_{r} \\
F_{t}
\end{array}\right]=\left[\begin{array}{c}
F_{r 0} \\
F_{t 0}
\end{array}\right]-\left[\begin{array}{ll}
C_{r r} & C_{r t} \\
C_{t r} & C_{t t}
\end{array}\right]\left(\begin{array}{c}
\Delta \dot{e} \\
e \Delta \dot{\varphi}
\end{array}\right)-\left[\begin{array}{cc}
K_{r r} & K_{r t} \\
K_{t r} & K_{t t}
\end{array}\right]\left(\begin{array}{c}
\Delta e \\
e \Delta \varphi
\end{array}\right)
$$

At this point, the terms $H_{0}=\frac{h_{0}}{c}$ and $H_{0}^{\prime}=\frac{h_{0}^{\prime}}{c}$ are introduced.

A first order Taylor series expansion was used to solve equation (11):

$$
H^{-3}=H_{0}^{-3}-3 \cdot H_{0}^{-4} \cdot H_{1}
$$

The radial and tangential forces acting on the journal, $F_{r 0}$ and $F_{t 0}$ respectively, can be calculated by substituting (10) into (11):

$$
\begin{aligned}
& F_{r 0}=\frac{\mu \cdot R \cdot L^{3}}{c^{3}} \cdot \frac{\Omega}{2} \cdot\left(\int_{0}^{\theta_{\varepsilon}} \frac{e_{0} \cdot \sin \theta \cdot \cos \theta}{H_{0}^{3}} d \theta+\int_{\theta_{s}}^{\theta_{f}}\left(\frac{e_{0} \cdot \sin \theta}{H_{0}^{\prime 3}}-\frac{c \cdot \sin \left(\theta+\varphi_{0}\right)}{H_{0}^{\prime 3}}\right) \cdot \cos \theta d \theta+\int_{\theta_{f}}^{\pi} \frac{e_{0} \cdot \sin \theta \cdot \cos \theta}{H_{0}^{3}} d \theta\right) \\
& F_{t 0}=\frac{\mu \cdot R \cdot L^{3}}{c^{3}} \cdot \frac{\Omega}{2} \cdot\left(\int_{0}^{\theta_{s}} \frac{e_{0} \cdot(\sin \theta)^{2}}{H_{0}{ }^{3}} d \theta+\int_{\theta_{s}}^{\theta_{f}}\left(\frac{e_{0} \cdot \sin \theta}{H_{0}^{\prime 3}}-\frac{c \cdot \sin \left(\theta+\varphi_{0}\right)}{H_{0}^{\prime 3}}\right) \cdot \sin \theta d \theta+\int_{\theta_{f}}^{\pi} \frac{e_{0} \cdot(\sin \theta)^{2}}{H_{0}{ }^{3}} d \theta\right)
\end{aligned}
$$

The dynamic coefficients of the bearing were calculated by solving equation (11) with respect to $K_{i j}$ and $C_{i j}$ :

$$
\begin{aligned}
& K_{r r}=\frac{\mu \cdot R \cdot L^{3}}{c^{3}} \cdot \frac{\Omega}{2} \cdot\left(\begin{array}{l}
-\int_{0}^{\theta_{i}} \frac{\sin \theta \cdot \cos \theta}{H_{0}{ }^{3}} d \theta+3 \cdot \varepsilon_{0} \cdot \int_{0}^{\theta_{i}} \frac{\sin \theta \cdot(\cos \theta)^{2}}{H_{0}{ }^{2}} d \theta-\int_{\theta_{f}}^{\pi} \frac{\sin \theta \cdot \cos \theta}{H_{0}{ }^{3}} d \theta+3 \cdot \varepsilon_{0} \cdot \int_{\theta_{f}}^{\pi} \frac{\sin \theta \cdot(\cos \theta)^{2}}{H_{0}{ }^{2}} d \theta- \\
-\int_{\theta_{s}}^{\theta_{f}} \frac{\sin \theta \cdot \cos \theta}{H_{0}{ }^{3}} d \theta+3 \cdot \varepsilon_{0} \cdot \int_{\theta_{s}}^{\theta_{f}} \frac{\sin \theta \cdot(\cos \theta)^{2}}{H_{0}^{\prime 4}} d \theta-3 \cdot \int_{\theta_{s}}^{\theta_{f}} \frac{(\cos \theta)^{2} \cdot \sin \left(\theta+\varphi_{0}\right)}{H_{0}^{\prime 4}} d \theta
\end{array}\right)(16 \\
& K_{r t}=\frac{\mu \cdot R \cdot L^{3}}{c^{3}} \cdot \frac{\Omega}{2} \cdot\left(\begin{array}{l}
\int_{0}^{\theta_{s}} \frac{(\cos \theta)^{2}}{H_{0}^{3}} d \theta+3 \cdot \varepsilon_{0} \cdot \int_{0}^{\theta_{s}} \frac{(\sin \theta)^{2} \cdot \cos \theta}{H_{0}^{4}} d \theta+\int_{\theta_{f}}^{\pi} \frac{(\cos \theta)^{2}}{H_{0}^{3}} d \theta+ \\
+3 \cdot \varepsilon_{0} \cdot \int_{\theta_{f}}^{\pi} \frac{(\sin \theta)^{2} \cdot \cos \theta}{H_{0}^{4}} d \theta+3 \cdot \varepsilon_{0} \cdot \int_{\theta_{s}}^{\theta_{f}} \frac{(\sin \theta)^{2} \cdot \cos \theta}{H_{0}^{\prime 4}} d \theta+ \\
+\int_{\theta_{s}}^{\theta_{f}} \frac{(\cos \theta)^{2}}{H_{0}^{\prime 3}} d \theta-3 \cdot \int_{\theta_{s}}^{\theta_{f}} \frac{\cos \theta \cdot \sin \theta \cdot \sin \left(\theta+\varphi_{0}\right)}{H_{0}^{\prime 4}} d \theta
\end{array}\right) \\
& K_{t r}=\frac{\mu \cdot R \cdot L^{3}}{c^{3}} \cdot \frac{\Omega}{2} \cdot\left(\begin{array}{l}
-\int_{0}^{\theta_{s}} \frac{(\sin \theta)^{2}}{H_{0}{ }^{3}} d \theta+3 \cdot \varepsilon_{0} \cdot \int_{0}^{\theta_{s}} \frac{(\sin \theta)^{2} \cdot \cos \theta}{H_{0}^{4}} d \theta-\int_{\theta_{f}}^{\pi} \frac{(\sin \theta)^{2}}{H_{0}{ }^{3}} d \theta+ \\
+3 \cdot \varepsilon_{0} \cdot \int_{\theta_{f}}^{\pi} \frac{(\sin \theta)^{2} \cdot \cos \theta}{H_{0}{ }^{4}} d \theta+3 \cdot \varepsilon_{0} \cdot \int_{\theta_{s}}^{\theta_{f}} \frac{(\sin \theta)^{2} \cdot \cos \theta}{H_{0}^{\prime 4}} d \theta- \\
-\int_{\theta_{s}}^{\theta_{f}} \frac{(\sin \theta)^{2}}{H_{0}^{\prime 3}} d \theta-3 \cdot \int_{\theta_{s}}^{\theta_{f}} \frac{\sin \theta \cdot \cos \theta \cdot \sin \left(\theta+\varphi_{0}\right)}{H_{0}^{\prime 4}} d \theta
\end{array}\right)
\end{aligned}
$$




$$
\begin{aligned}
& K_{t t}=\frac{\mu \cdot R \cdot L^{3}}{c^{3}} \cdot \frac{\Omega}{2} \cdot\left(\begin{array}{l}
\int_{0}^{\theta_{i}} \frac{\sin \theta \cdot \cos \theta}{H_{0}^{3}} d \theta+3 \cdot \varepsilon_{0} \cdot \int_{0}^{\theta_{\delta}} \frac{(\sin \theta)^{3}}{H_{0}^{4}} d \theta+\int_{\theta_{f}}^{\pi} \frac{\sin \theta \cdot \cos \theta}{H_{0}^{3}} d \theta+ \\
+3 \cdot \varepsilon_{0} \cdot \int_{\theta_{f}}^{\pi} \frac{(\sin \theta)^{3}}{H_{0}^{4}} d \theta+3 \cdot \varepsilon_{0} \cdot \int_{\theta_{s}}^{\theta_{f}} \frac{(\sin \theta)^{3}}{H_{0}^{\prime 4}} d \theta+\int_{\theta_{s}}^{\theta_{f}} \frac{\sin \theta \cdot \cos \theta}{H_{0}^{\prime 3}} d \theta \\
-3 \cdot \int_{\theta_{s}}^{\theta_{f}} \frac{(\sin \theta)^{2} \cdot \sin \left(\theta+\varphi_{0}\right)}{H_{0}^{\prime 4}} d \theta
\end{array}\right) \\
& C_{r r}=\frac{\mu \cdot R \cdot L^{3}}{c^{3}} \cdot\left(\int_{0}^{\theta_{s}} \frac{(\cos \theta)^{2}}{H_{0}{ }^{3}} d \theta+\int_{\theta_{s}}^{\theta_{f}} \frac{(\cos \theta)^{2}}{H_{0}^{\prime 3}} d \theta+\int_{\theta_{f}}^{\pi} \frac{(\cos \theta)^{2}}{H_{0}{ }^{3}} d \theta\right) \\
& C_{r t}=\frac{\mu \cdot R \cdot L^{3}}{c^{3}} \cdot\left(\int_{0}^{\theta_{i}} \frac{\sin \theta \cdot \cos \theta}{H_{0}{ }^{3}} d \theta+\int_{\theta_{s}}^{\theta_{f}} \frac{\sin \theta \cdot \cos \theta}{H_{0}^{\prime 3}} d \theta+\int_{\theta_{f}}^{\pi} \frac{\sin \theta \cdot \cos \theta}{H_{0}{ }^{3}} d \theta\right) \\
& C_{t r}=\frac{\mu \cdot R \cdot L^{3}}{c^{3}} \cdot\left(\int_{0}^{\theta_{i}} \frac{\sin \theta \cdot \cos \theta}{H_{0}{ }^{3}} d \theta+\int_{\theta_{s}}^{\theta_{f}} \frac{\sin \theta \cdot \cos \theta}{H_{0}^{\prime 3}} d \theta+\int_{\theta_{f}}^{\pi} \frac{\sin \theta \cdot \cos \theta}{H_{0}{ }^{3}} d \theta\right) \\
& C_{t t}=\frac{\mu \cdot R \cdot L^{3}}{c^{3}} \cdot\left(\int_{0}^{\theta_{s}} \frac{(\sin \theta)^{2}}{H_{0}^{3}} d \theta+\int_{\theta_{s}}^{\theta_{f}} \frac{(\sin \theta)^{2}}{H_{0}^{\prime 3}} d \theta+\int_{\theta_{f}}^{\pi} \frac{(\sin \theta)^{2}}{H_{0}^{3}} d \theta\right)
\end{aligned}
$$

The dynamic coefficients listed above, refer to the rotating coordinate system and not to the Cartesian coordinate system. The relationship between the dynamic coefficients in both coordinate systems can be determined by the following transformations:

$$
\begin{aligned}
& {\left[\begin{array}{ll}
K_{x x} & K_{x y} \\
K_{y x} & K_{y y}
\end{array}\right]=\left[\begin{array}{cc}
\cos \left(\varphi_{0}\right) & -\sin \left(\varphi_{0}\right) \\
\sin \left(\varphi_{0}\right) & \cos \left(\varphi_{0}\right)
\end{array}\right]\left[\begin{array}{ll}
K_{r r} & K_{r t} \\
K_{t r} & K_{t t}
\end{array}\right]\left[\begin{array}{cc}
\cos \left(\varphi_{0}\right) & \sin \left(\varphi_{0}\right) \\
-\sin \left(\varphi_{0}\right) & \cos \left(\varphi_{0}\right)
\end{array}\right]} \\
& {\left[\begin{array}{ll}
C_{x x} & C_{x y} \\
C_{y x} & C_{y y}
\end{array}\right]=\left[\begin{array}{cc}
\cos \left(\varphi_{0}\right) & -\sin \left(\varphi_{0}\right) \\
\sin \left(\varphi_{0}\right) & \cos \left(\varphi_{0}\right)
\end{array}\right]\left[\begin{array}{ll}
C_{r r} & C_{r t} \\
C_{t r} & C_{t t}
\end{array}\right]\left[\begin{array}{cc}
\cos \left(\varphi_{0}\right) & \sin \left(\varphi_{0}\right) \\
-\sin \left(\varphi_{0}\right) & \cos \left(\varphi_{0}\right)
\end{array}\right]}
\end{aligned}
$$

\section{Finite element solution}

A script in the ANSYS software was created for the calculation of the dynamic coefficients of a nonworn journal bearing. The functions of the script are presented in the following lines.

- Deletion of old data.

- Determination of the size of the matrices in which the results (equilibrium position \& dynamic coefficients) were going to be stored.

- Mesh consisted of 180 divisions on the circumferential direction, 10 divisions across the bearing's oil film and 10 divisions in the axial direction.

- Coordinate systems (Cartesian \& rotational) situated on the center of the rotor and the bearing.

- Computation of the linear velocities of the nodes of the rotor.

- Computation of the film pressure and the forces acting on the rotor.

- Calculation of the equilibrium position with a loop which implemented the 2-D NewtonRaphson search technique. This loop was ended when the desired accuracy was achieved.

- Computation of the stiffness and damping dynamic coefficients according to the following equations: 


$$
\begin{gathered}
K_{i j}=-\frac{\partial F_{i}}{\partial x_{j}} \\
C_{i j}=-\frac{\partial F_{i}}{\partial \dot{x}_{j}}
\end{gathered}
$$

\section{Results}

In this paragraph, the results related to the dynamic coefficients of the analytical method and the finite element model are presented.

\subsection{Analytical solution}

As for the analytical solution, figures 2 and 3 depict the dimensionless stiffness and damping dynamic coefficients respectively, versus the modified Sommerfeld number, for a non-worn journal bearing.

In short length journal bearings, the modified Sommerfeld number $\sigma$ is defined as:

$$
\sigma=\pi S\left(\frac{L}{D}\right)^{2}=\frac{\mu \Omega L R}{4 W}\left(\frac{L}{c}\right)^{2}
$$

The geometrical and operational characteristics of the bearing, considered for the present analysis, are:

- Radial clearance $c=20 \times 10^{-6} \mathrm{~m}$

- Bearing radius $R=25 \times 10^{-3} \mathrm{~m}$

- Bearing axial length $L=12.5 \times 10^{-3} \mathrm{~m}$

- Journal rotational speed $\Omega=300 \mathrm{rad} / \mathrm{s}$

- Absolute oil viscosity $\mu=0.012$ Pa.s

In figure 2, the negative values of the $K_{y x}$ dynamic stiffness coefficient have been replaced by their absolute values in order to be represented in a logarithmic diagram.

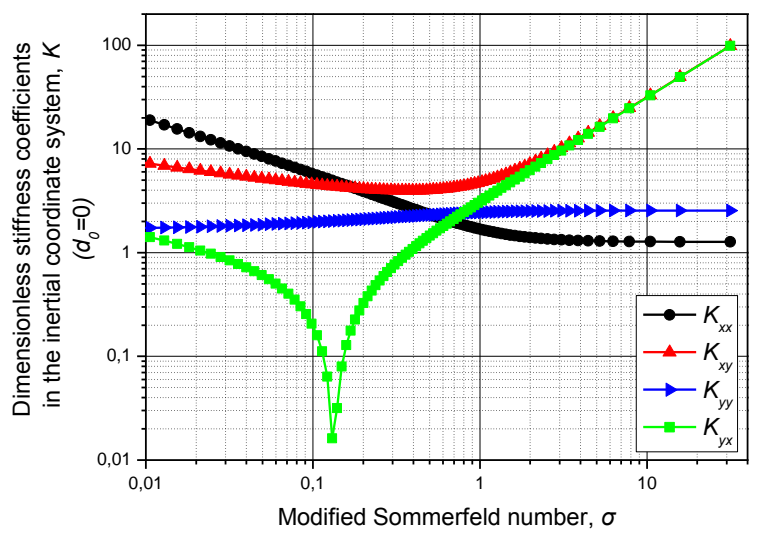

Figure 2: Dimensionless stiffness dynamic coefficients vs. modified Sommerfeld number

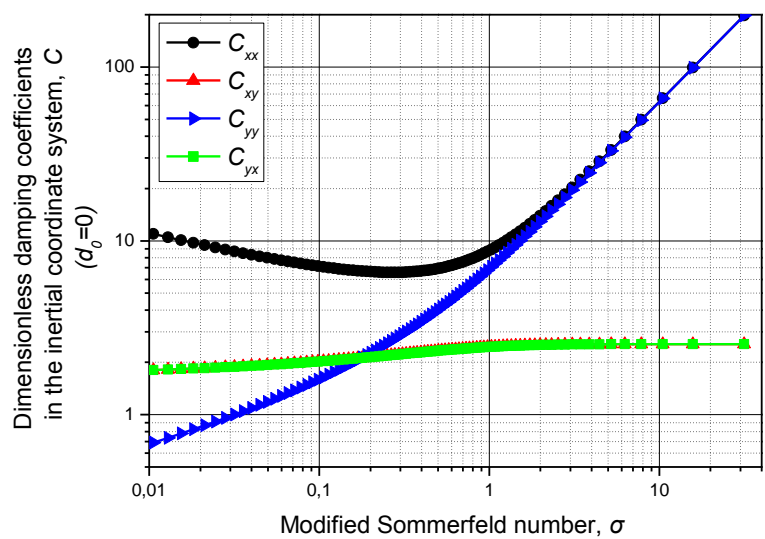

Figure 3: Dimensionless damping dynamic coefficients vs. modified Sommerfeld number

The effect of the geometric change due to wear, on the dimensionless stiffness and damping dynamic coefficients of a bearing is depicted in figures 4 and 5 (modified Sommerfeld number $\sigma=0.185)$. More specifically, the coefficients over the ones corresponding to the intact case $\left(d_{0}=0\right)$ are presented. 


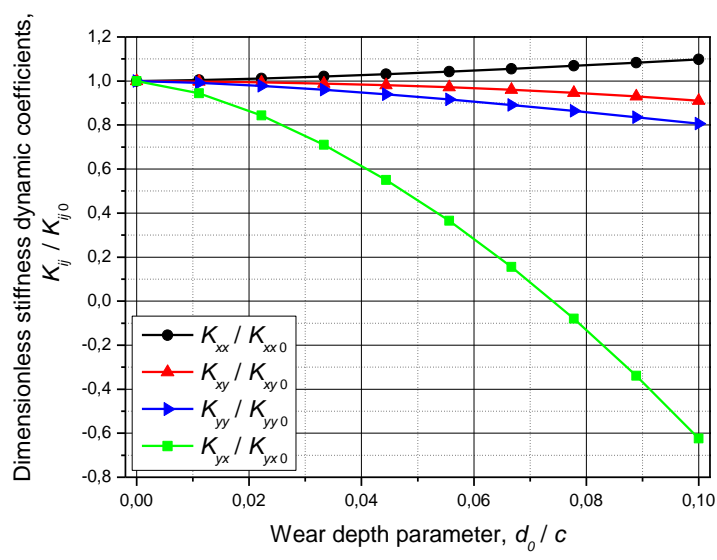

Figure 4: Dimensionless stiffness dynamic coefficients vs. wear depth parameter

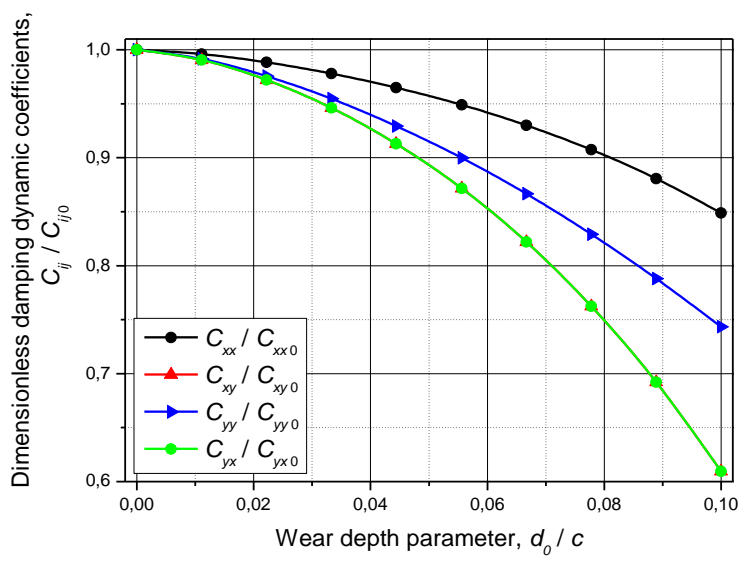

Figure 5: Dimensionless damping dynamic coefficients vs. wear depth parameter

\subsection{Finite element solution}

In this paragraph, the results of the finite element solution are presented. The geometrical and operational characteristics of the bearing for the FEM analysis are identical with those used in the analytical approach.

For the purposes of the current analysis, a dynamic mesh was designed. Specifically, the nodes of the mesh were not static, but they could adjust their position analogically, to obtain more accurate results. The mesh is depicted in figures 6 and 7.

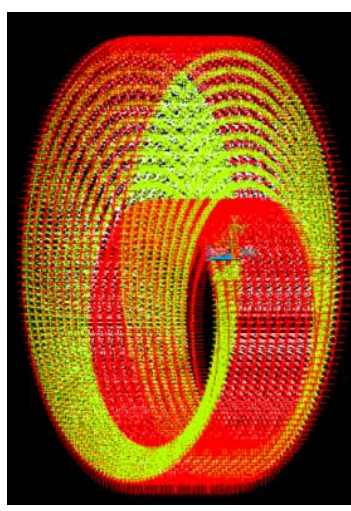

Figure 6: Mesh of a non-worn journal bearing

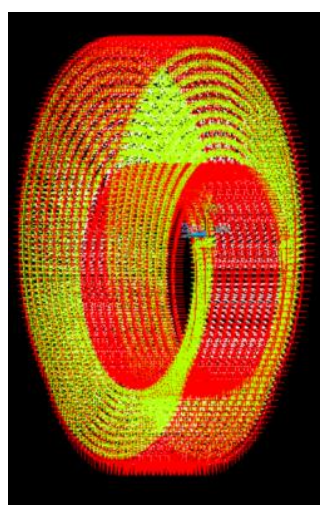

Figure 7: Mesh of a worn journal bearing

The pressure distribution on the circumference of a non-worn and a worn journal bearing respectively is depicted in figures 8 and 9 .

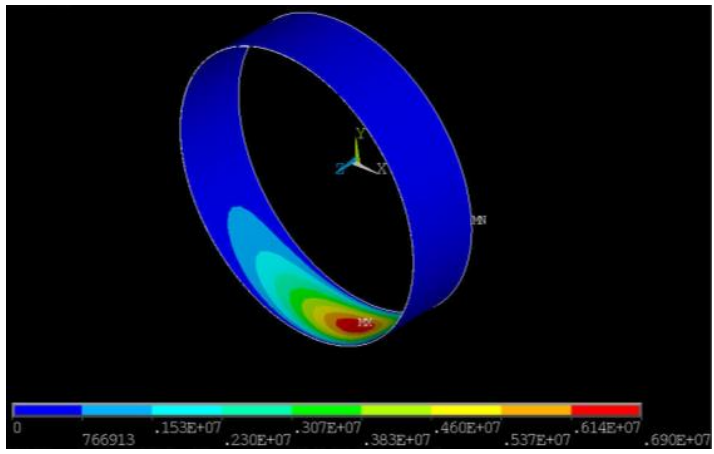

Figure 8: Pressure distribution on the circumference of a non-worn journal bearing

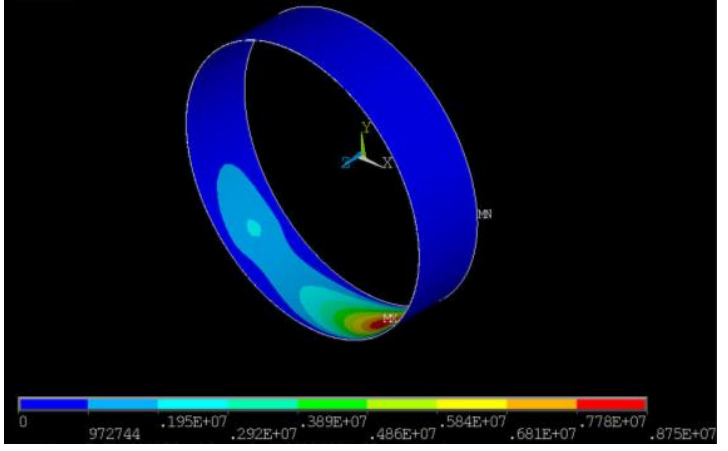

Figure 9: Pressure distribution on the circumference of a worn journal bearing

Figure 10 illustrates the variation of the position of the equilibrium point, as a function of the Sommerfeld number and the wear depth parameter. 


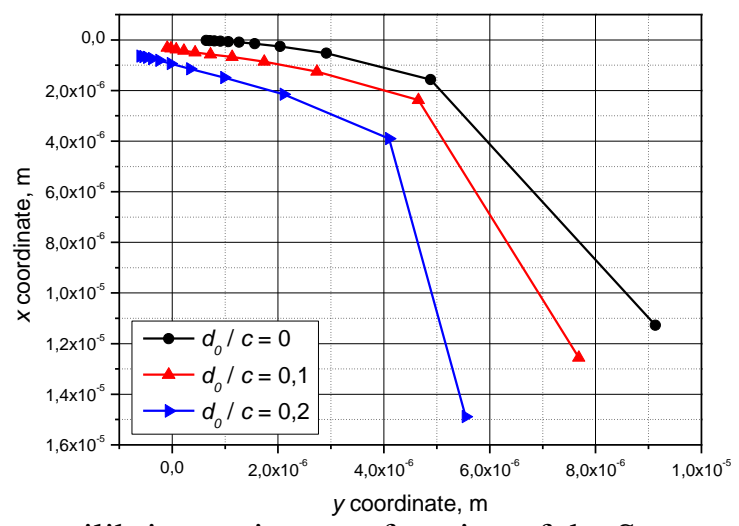

Figure 10: Variation of the equilibrium point, as a function of the Sommerfeld number and the wear depth parameter

Figures 11 and 12 present the dimensionless stiffness and damping dynamic coefficients respectively versus the modified Sommerfeld number, for a non-worn journal bearing, via the FEM solution.

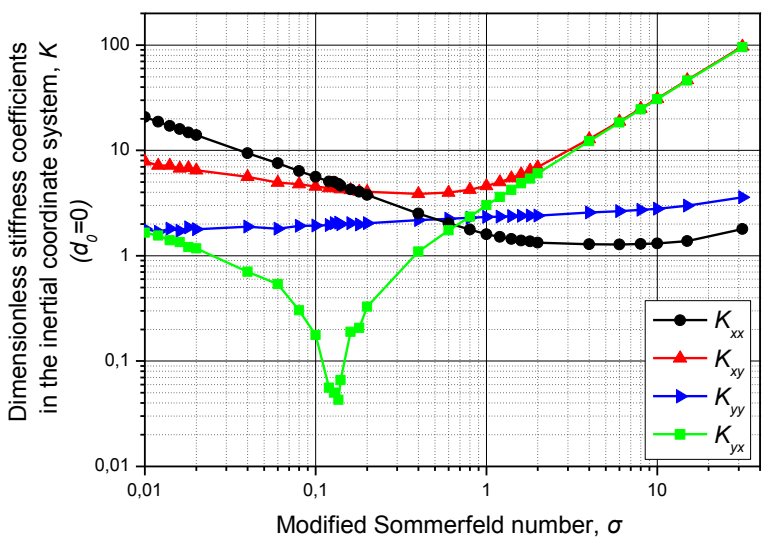

Figure 11: Dimensionless stiffness dynamic coefficients vs. modified Sommerfeld number

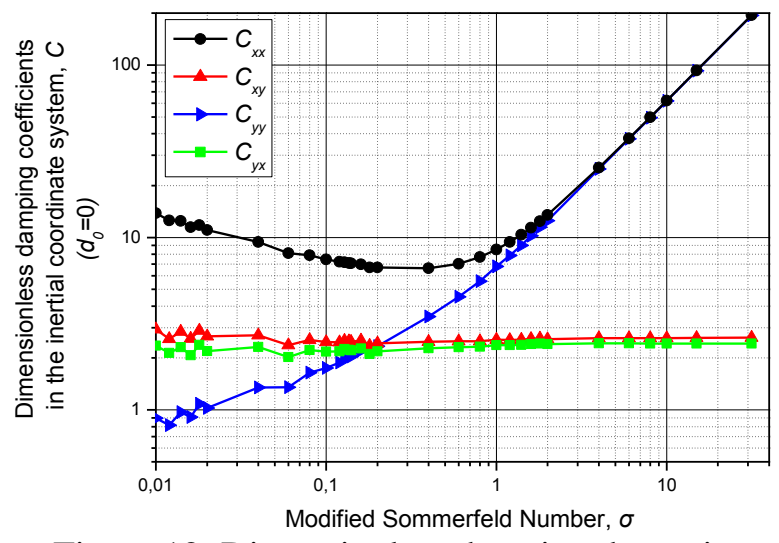

Figure 12: Dimensionless damping dynamic coefficients vs. modified Sommerfeld number

The effect of the geometric change due to wear, on the dimensionless stiffness and damping dynamic coefficients of a bearing, is depicted in figures 13 and 14 (modified Sommerfeld number $\sigma=0.185)$.

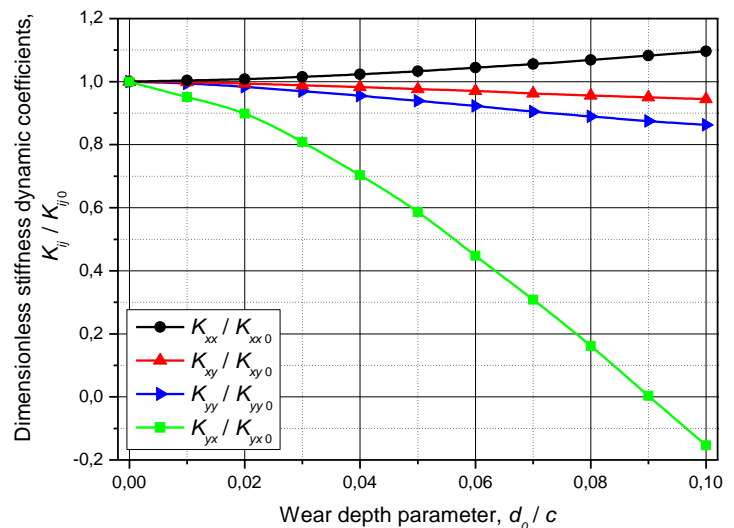

Figure 13: Dimensionless stiffness dynamic coefficients vs. wear depth parameter

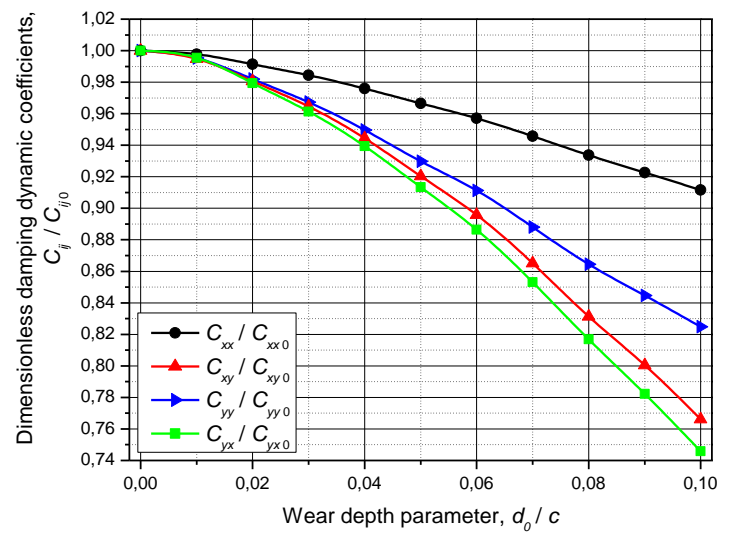

Figure 14: Dimensionless damping dynamic coefficients vs. wear depth parameter

The differences in the results between the semi-analytical and the FEM results exist due to the following reasons:

- The semi-analytical solution is based on the assumption that eccentricity and attitude angle are 
linear functions $\left(e(t)=e_{0}+\Delta e(t), \varphi(t)=\varphi_{0}+\Delta \varphi(t)\right)$, which does not reflect reality for large rotor displacements.

- The results obtained from the semi analytical solution (figures 4 and 5), do not refer to a constant modified Sommerfeld number (modified Sommerfeld number alters slightly while the bearing's geometry changes). In contrast, results obtained by the FEM solution (figures 13 and 14) refer to a constant modified Sommerfeld number.

- The Reynolds equation used in the semi-analytical solution has been simplified using a Taylor series, to describe the properties of infinitely short journal bearings (L/D0), while the bearing studied in this project is not infinitely short $(\mathrm{L} / \mathrm{D}>0)$. Therefore, deviations from the correct results can be justified.

\section{Conclusions}

To conclude, the analytical solution results are not identical to those produced by the FEM analysis. However, it can be seen that there are many similarities between the results of the two solutions. Therefore, the analytical solution could be trusted, especially when the wear depth parameter is small and the computational cost should be reduced. There is deviation of the analytical solution from the expected results (FEM solution), because of the assumptions made for the analytical solution, which affect the results, especially for high $L / D$ ratios.

\section{References}

1. Mokhtar, M., Howarth, R., and Davies, P., Wear Characteristics of Plain Hydrodynamic Journal Bearings During Repated Starting and Stopping, ASLE Trans.(1977) 20 191-194.

2. Kumar A. and Mishra S.S., Stability of rigid rotor in turbulent hydrodynamic worn journal bearings, Wear, (1996) 193: 25-30

3. Kumar A. and Mishra S.S., Steady state analysis of non-circular worn journal bearings in nonlaminar lubrication regimes, Tribology International, (1996) 29 (6) 493-498.

4. Laurant F. and Childs D.W., Measurement of rotordynamic coefficients of hybrid bearings with (a) plugged orifice (b) a worn land surface, Journal of Engineering for Gas Turbines and Power, (2002) 124: 363-368.

5. Awasthi R.K., Sharma S.C. and Jain S.C., Performance of worn non-recessed hole-entry hybrid journal bearings, Tribology International, (2007) 40: 717-734.

6. Vaidyanathan K., and Keith T.G., Performance characteristics of cavitated non-circular journal bearings in the turbulent flow regime, Tribology Transactions, (1991) 34: 35-44

7. Hashimoto H., Wada S. and Nojima K., Performance Characteristics of Worn Journal Bearings in Both Laminar and Turbulent Regimes. Part II: Dynamic Characteristics, ASLE transactions, (1986) 29 (4) $572-577$.

8. Chasalevris A.C., Nikolakopoulos P.G. and Papadopoulos C.A., Dynamic effect of bearing wear on rotor-bearing system response, Journal of Vibration and Acoustics, (2013) 135 (1) 1-12. (http://dx.doi.org/10.1115/1.4007264)

9. Gertzos K.P., Nikolakopoulos P.G., Chasalevris A.C. and Papadopoulos C.A., Wear identification in rotor-bearing systems by measurements of dynamic bearing characteristics, Computers and Structures, (2011) 89 (1-2) 55-66.

10.Rozeanu L. and Kennedy F.E., Wear of hydrodynamic journal bearings, Tribology Series, (2001) 39 161-166.

11.Ronen A. and Malkin S. Wear mechanisms of statically loaded hydrodynamic bearings by contaminant abrasive particles, Wear, (1981) 68 (3) 371-389.

12.Li Y., Zhu L., Wang J., Gui H., Yan D. and Huang, Q. Study on the Wear Properties of Hydrodynamic Oil Film Bearing by Finite Element Method, Applied Mechanics and Materials, (2010) 37 - 38 1356-1359, DOI 10.4028/www.scientific.net/AMM.37-38.1356.

13. Bouyer J., Fillon M. and Pierre-Danos I. Influence of wear on the behavior of a two-lobe hydrodynamic journal bearing subjected to numerous startups and stops, Journal of Tribology, (2007) 129 (1) 205-208.

14.Hamrock B.J., Schmid S.R. and Jacobson B.O., Fundamentals of Fluid Film Lubrication, $2^{\text {nd }}$ edition, Marcel Dekker Inc, New York 2004. [1]

15.Phalle V.M., Sharma S.C. and Jain S.C., Influence of wear on the performance of a 2-lobe multirecess hybrid journal bearing system compensated with membrane restrictor, Tribology 
International, (2011) 44 380-395.

16.Scharrer J.K., Hecht R.F. and Hibbs R.I., The effects of wear on the rotordynamic coefficients of hydrostatic journal bearings, Journal of Tribology, (1991) 113: 210-213.

17.Nikolakopoulos P. and Papadopoulos C.A., A study of friction in worn misaligned journal bearings under severe hydrodynamic lubrication, Tribology International, (2008) 41: 461-472.

18.Dufrane K.F., Kannel J.W. and McCloskey T.H., Wear of steam turbine Journal bearings at low operating speeds, Journal of Lubrication Technology, Transactions of ASME, (1983) 105 (3) 313317. 


\title{
Alternation of the dynamic coefficients of short journal bearings due to wear
}

\author{
Papanikolaou, Michail
}

\section{Emerald}

Michael G. Papanikolaou, Michael G. Farmakopoulos and Chris A. Papadopoulos. Alternation of the dynamic coefficients of short journal bearings due to wear. International Journal of Structural Integrity, Volume 6, Issue 5, 2015, pp. 649-664

https://doi.org/10.1108/lJSI-05-2014-0025

Downloaded from Cranfield Library Services E-Repository 